\title{
Epidemiology, etiology, and treatment of denture stomatitis
}

\author{
Meisan Ali Bukhari ${ }^{1 *}$, Mashael Abdullah Algahtani², Faisal Abdullah Alsuwailem ${ }^{3}$, \\ Rakan Mishaal Alogaiel ${ }^{4}$, Safa Hejji Almubarak ${ }^{5}$, Saud Salman Alqahtani ${ }^{6}$, \\ Raghdah Abdulaziz Alabdullatif ${ }^{7}$, Reem Yaqoub Alghimlas ${ }^{8}$, Noura Fahad Alotaibi ${ }^{9}$, \\ Atheer Rakkad Al Qahtani ${ }^{10}$, Norah Khaled Alkathiri ${ }^{10}$
}

\author{
${ }^{1}$ North Jeddah Specialist Dental Center, King Abdullah Medical Complex, Jeddah, Saudi Arabia \\ ${ }^{2}$ Taif Specialized Dental Center, Ministry of Health, Taif, Saudi Arabia \\ ${ }^{3}$ College of Dentistry, King Saud University, Riyadh, Saudi Arabia \\ ${ }^{4}$ College of Dentistry, Imam Abdulrahman Bin Faisal University, Dammam, Saudi Arabia \\ ${ }^{5}$ Ministry of Health, Turaif, Saudi Arabia \\ ${ }^{6}$ College of Dentistry, King Faisal University, Hofuf, Saudi Arabia \\ ${ }^{7}$ Primary Healthcare Center, Ministry of Health, Buraydah, Saudi Arabia \\ ${ }^{8}$ College of Dentistry, Princess Nourah Bint Abdul Rahman University, Riyadh, Saudi Arabia \\ ${ }^{9}$ General Dentist, Prince Sultan Military Medical City, Riyadh, Saudi Arabia \\ ${ }^{10}$ College of Dentistry, Vision Colleges, Riyadh, Saudi Arabia
}

Received: 12 December 2021

Accepted: 27 December 2021

\section{*Correspondence: \\ Dr. Meisan Ali Bukhari, \\ E-mail: meisan-aml@gmail.com}

Copyright: (C) the author(s), publisher and licensee Medip Academy. This is an open-access article distributed under the terms of the Creative Commons Attribution Non-Commercial License, which permits unrestricted non-commercial use, distribution, and reproduction in any medium, provided the original work is properly cited.

\begin{abstract}
Multiple factors are involved in the pathogenesis of denture stomatitis, which increases the risk of tissue infection and inflammation. These factors include poor oral hygiene, trauma secondary to poorly fitting prostheses, resin porosity, and bacterial plaque accumulation. Our present review discusses the epidemiology, etiology, and treatment of denture stomatitis based on data from current studies in the literature. The prevalence of denture stomatitis is significantly variable among the different studies, as previously discussed. However, the cumulative incidence of denture stomatitis among their participants ranged between $17-77 \%$. These hugely variable rates have been attributed to the nature of data collection, diagnostic criteria, sample size, and patient demographics. Studies also show that the condition is more prevalent among elderly females. However, not many studies have reported this correlation, indicating the need for future studies. Candida albicans infection is the primary parameter in the etiology and pathogenesis of the condition. However, other factors related to the patient (like status of immunological response) and dentures (like hygiene) were also reported. Therefore, the management of denture stomatitis should be based on applying adequate interventions. Besides, using antifungal medications is also necessary to eradicate organism.
\end{abstract}

Keywords: Denture stomatitis, Candida albicans, Oral infection, Prosthesis

\section{INTRODUCTION}

Estimates show that denture stomatitis is among the most commonly reported disorder among dental prosthesis users. ${ }^{1}$ Multiple factors are involved in the pathogenesis of denture stomatitis, which increases the risk of tissue infection and inflammation. These factors include poor oral hygiene, trauma secondary to poorly fitting prostheses, resin porosity, and bacterial plaque accumulation. $^{2,3}$ About two-thirds of upper complete denture users suffer from this condition. Most of the affected patients are usually asymptomatic, and the condition mostly occurs in the palatine mucosa. However, it should be noted that some patients might have some 
clinical manifestations. These include burning sensations, pain, halitosis, and pruritis. ${ }^{4}$

The diagnosis of denture stomatitis is mainly dependent on clinical manifestations. These include the presence of edema and erythema on the palatal gingiva and mucosa covered by denture base. ${ }^{5}$ In addition, infections by Candida albicans are usually associated with many cases. Therefore, further workup might be needed when this etiology has been determined. ${ }^{6,7}$ It should be noted that the epidemiology and etiology of the condition are inconsistent among different studies. Accordingly, we will discuss the epidemiology, etiology, and treatment of denture stomatitis in a comprehensive literature review.

\section{LITERATURE REVIEW}

This literature review is based on an extensive literature search in Medline, Cochrane, and EMBASE databases on which was performed $3^{\text {rd }}$ December 2021 using the medical subject headings (MeSH) or a combination of all possible related terms, according to the database. To avoid missing poetential studies, a further manual search for papers was done through Google Scholar, while the reference lists of the initially included papers. Studies discussing Epidemiology, etiology, and treatment of denture stomatitis were screened for useful information, with no limitations posed on date, language, age of participants, or publication type.

\section{DISCUSSION}

\section{Epidemiology}

Many previous epidemiological studies have assessed the prevalence of denture stomatitis within different global settings. Among the various studies in the literature, estimates show that the prevalence of denture stomatitis among different adult populations ranges between 15$71 \%$. These studies recruited patients from long-term care facilities, nursing homes, and community settings. For example, studies in Turkey, Spain, Slovenia, and Denmark reported that the prevalence of denture stomatitis in their population was $18.5 \%, 19.6 \%, 14.7 \%$, and $65 \%$, respectively, among denture wearers. ${ }^{8-11}$ The study conducted in Denmark and reported high prevalence rate showed that this is attributed to the high prevalence of correlated candida infection and bad oral hygiene in their elderly population. ${ }^{11}$ Another 2 population-based studies were also conducted in Finland. One of these studies included home-living elderly residents using a stratified sampling technique, while the other 1 included a national sample. The authors reported that the prevalence of denture stomatitis among the two studies was $35 \%$ and $48 \%$, respectively. ${ }^{12,13}$ The Finnish national-based survey indicated that around $44 \%$ of adults in Finland have denture stomatitis. Another nationalbased investigation was also conducted in Germany and reported different rates of denture stomatitis for different age groups. The authors estimated that the prevalence of condition was $18.3 \%$ and $2.5 \%$ for participants aged 65 74 and 34-44 years old, respectively. ${ }^{14}$

A previous study in Denmark aimed to estimate the prevalence of denture stomatitis among elderly denture wearers that were recruited from long-term care facilities and nursing settings and found that the prevalence was $34 \% .{ }^{15}$ On the other hand, another study reported that the prevalence of the condition was as twice as much as the reported prevalence in this study among communitydwelling elderly. Furthermore, previous studies in Finland also reported that the prevalence of denture stomatitis among institutionalized and home-living denture wearers was $25 \%$ and $35 \%$, respectively. Other investigations were also conducted in South America. For instance, two previous studies in Brazil reported that the prevalence of denture stomatitis among elderly denture wearers in long-term care facilities was $54 \%-58.2 \% .^{16,17}$

On the other hand, another study from Chile reported that the prevalence of the condition was $34.5 \%$. Other investigations in the literature assessed the prevalence of denture stomatitis among patients visiting prosthetic and dental clinics for replacement, adjustment, or treatment of dentures. These studies were conducted in different global communities, and cumulative incidence of denture stomatitis among their participants ranged between 17$77 \%$. It has been suggested that the different sample sizes among the included studies were a significant factor for the different prevalence rates among these studies. ${ }^{18}$

Other studies also assessed the prevalence of denture stomatitis among men and women. For instance, the two previous Finnish investigations reported that the condition's prevalence was significantly higher among females. This has also been indicated in another investigation in Slovenia. In addition, another study in Chile reported that the prevalence of denture stomatitis was also lower among males than females $(25.1 \%$ versus $38.7 \%$, respectively). ${ }^{1,18}$ However, it should be noted that not all of included population-based investigations reported the potential differences in prevalence of denture stomatitis between males and females. For instance, other studies from Brazil reported no significant association between gender of included participants and prevalence of denture stomatitis. In addition, it should be noted that some studies might have had limitations regarding sample size and data collection, which might have significantly influenced their outcomes. Therefore, further studies might still be needed for further elaboration. ${ }^{18}$

\section{Etiology}

The pathogenesis of denture stomatitis is mostly attributed to Candida albicans infection. ${ }^{4}$ The organism is naturally present within the mouth. However, certain conditions might turn it into pathogenic and cause the disease. Severe factors were reported in this context. These include malignant diseases, immunological and endocrine disorders, diabetes mellitus, HIV, 
immunosuppression, xerostomia, poor oral hygiene, tissue trauma secondary to inadequate occlusion, and continuous use of prostheses. Accordingly, the organism can invade oral tissues and break protective barriers of the oral mucosa. ${ }^{19-21}$ However, it should be noted that different studies in the literature and previous reviews have concluded that the etiology of denture stomatitis is multifactorial. ${ }^{22}$ Some studies also concluded that some risk factors might be associated with the patient's denture use. These include lack of appropriate denture hygiene, using mandibular versus maxillary dentures, increasing age of the used dentures, and induced inflammation and trauma secondary to wearing unfit dentures. Allergy from denture materials was also reported as a potential factor playing a role in the pathogenesis of denture stomatitis.

In this context, it has been demonstrated that bad oral hygiene is a crucial risk factor for the development of denture stomatitis. Furthermore, poor oral hygiene has been associated with different practices. For instance, some studies reported that poor oral hygiene is significantly associated with wearing prostheses while sleeping. In the same context, a previous study reported that increased severity of inflammation, greater mucosal trauma, and increased Candida colonization of oral mucosa and dentures are significant events associated with poor oral hygiene. Some studies also emphasized the role of denture structure and materials in developing denture stomatitis. ${ }^{1}$ The primary role that has been reported in the literature is the ability of organisms within the oral cavity to form a biofilm over these structures. Poor denture fit and associated trauma and inflammation were also reported to predispose to developing denture stomatitis significantly. Moreover, various demographic factors might be significantly associated with the pathogenesis and severity of denture stomatitis. These include compromised immune functions, the presence of comorbidities, smoking, female gender, and increased duration of wearing dentures. ${ }^{18}$

\section{Treatment}

Evidence indicates that poor oral hygiene is the most significant factor associated with the development of denture stomatitis. However, studies also show that other factors might be associated, including reduced salivary flow, smoking, poor denture adaptation and high carbohydrate diets. In addition, it has been furtherly demonstrated that localized Newton's type I inflammation might also be associated with around $50 \%$ of denture wearers with poor filling dentures. ${ }^{10,23,24}$ Accordingly, it has been demonstrated that to increase the efficacy of prevention against denture stomatitis, further efforts should be exerted to enhance stability, retention, and support of removable dentures. Accordingly, it has been suggested that taking adequate care of oral hygiene is important in achieving adequate treatment of denture stomatitis. In this context, previous investigations reported that the quality of used prosthesis is important in intervening against the development of denture stomatitis and not methods used. ${ }^{19,25,26}$ Therefore, many protocols were published to enhance quality of cleaning in this regard. ${ }^{26,27}$ However, it has been shown that anti-fungal treatment is essential to enhance adequate treatment. ${ }^{28-32}$

A previous report showed that miconazole had been associated with favorable outcomes when treating denture stomatitis. ${ }^{33}$ However, evidence shows that the medication should be applied following proper hygiene approaches over the prothesis of the affected patients. Accordingly, the medication would be giving more contact with the lesion, which can significantly enhance healing and promote healing. In addition, it has been shown that systemic anti-fungal modalities should be furtherly administered when there are no clinical signs of improvement. $^{34,35}$ An $89 \%$ positive treatment response was also reported following the oral application of fluconazole (50 mg/day for 14 days). ${ }^{36}$

On the other hand, these studies demonstrated that the response rate among placebo patients was very minimal. However, it has been reported that after four weeks of treatment, some cases relapsed. Accordingly, it has been concluded that other topical anti-fungal modalities might be more efficacious than fluconazole oral application. On the other hand, a previous study by Martin-Mazuelos et al reported a significant regression in microbiological (78\%) and clinical $(97 \%)$ signs following the application of fluconazole. ${ }^{37}$ Moreover, the authors reported that patients with recurrence after treatment were treated with itraconazole (100 mg for 18 days). This has been associated with a $77 \%$ and $100 \%$ improvement in these patients' microbiological and clinical signs.

Previous investigations also compared the efficacy of itraconazole and fluconazole in reducing mycological cultures and palatal erythema. ${ }^{31,38,39}$ It has been found that the efficacy of both modalities was similar among different studies. However, it has been furtherly reported that some cases of disease persistence and organism recolonization were reported among these studies. Nystatin was also reported as an acceptable modality for managing fungal-related fungal stomatitis. ${ }^{40}$ Using tables and suspensions of this modality for two weeks was also associated with enhanced treatment outcomes. In addition, a significant improvement in the clinical signs was observed when nystatin was combined with amphotericin B. ${ }^{41}$ A previous study by Falah-Tafti et al compared the efficacy of fluconazole and nystatin and reported that treatment outcomes of denture stomatitis were more significant with nystatin, which has more abilities in reducing fungal colonization and binding. ${ }^{42}$

Moreover, it has been shown that the cost of the drug is not high, and the rate of nystatin fungi resistance is low compared to other anti-fungal medications. ${ }^{43}$ Ketoconazole was also described among previous investigations as a potential treatment for denture stomatitis. However, it has been shown that the systemic administration of this drug is usually associated with 
some adverse events. These include fever, abnormal tiredness, vomiting, nausea, and poor appetite. ${ }^{44}$ The evidence further demonstrated that topical application of clotrimazole is usually associated with favorable outcomes when treating denture stomatitis. ${ }^{45}$

Mouthwash-based modalities were also reported in the literature. For instance, chlorhexidine digluconate $(0.12 \%)$ was used to treat denture stomatitis and eliminated Candida albicans from the affected patients. It has been furtherly reported that this drug might also eradicate essential enzymes involved in the pathogenesis of fungal infections. ${ }^{28,46}$ In addition, candida-induced stomatitis can also be effectively managed by a novel modality called photodynamic therapy. In this context, it has been reported that the most commonly used photosensitizers include porphyrin derivatives, toluidine blue, and methylene blue. ${ }^{47-49}$ Further studies also evaluated the efficacy of nanomaterials in preventing denture stomatitis among susceptible patients. ${ }^{50}$ Findings from the majority of relevant investigations show that all of the proposed anti-fungal modalities are usually effective in managing denture stomatitis, with variable clinical and microbiological outcomes. However, most of these modalities are generally accepted for managing candida-based denture stomatitis.

\section{CONCLUSION}

The prevalence of denture stomatitis is significantly variable among the different studies, as previously discussed. These hugely variable rates have been attributed to the nature of data collection, diagnostic criteria, sample size, and patient demographics. Studies also show that the condition is more prevalent among elderly females. However, not many studies have reported this correlation, indicating the need for future studies. Candida albicans infection is the primary parameter in the etiology and pathogenesis of the condition. However, other factors related to the patient (like the status of immunological response) and dentures (like hygiene) were also reported. Therefore, the management of denture stomatitis should be based on applying adequate interventions. Besides, using anti-fungal medications is also necessary to eradicate the organism.

Funding: No funding sources Conflict of interest: None declared

Ethical approval: Not required

\section{REFERENCES}

1. Cubera K. Denture stomatitis-definition, etiology, classification and treatment. Przeglad lekarski. 2013;70(11):947-9.

2. Dhamande MM, Pakhan AJ, Thombare RU, Ghodpage SL. Evaluation of efficacy of commercial denture cleansing agents to reduce the fungal biofilm activity from heat polymerized denture acrylic resin:
An in vitro study. Contemporary clinical dentistry. 2012;3(2):168-72.

3. Hong G, Murata H, Li Y, Sadamori S, Hamada T. Influence of denture cleansers on the color stability of three types of denture base acrylic resin. J prosthetic dentistry. 2009;101(3):205-13.

4. Altarawneh S, Bencharit S, Mendoza L. Clinical and histological findings of denture stomatitis as related to intraoral colonization patterns of Candida albicans, salivary flow, and dry mouth. Journal of prosthodontics : official journal of the American College of Prosthodontists. 2013;22(1):13-22.

5. Aoun G, Berberi A. Prevalence of Chronic Erythematous Candidiasis in Lebanese Denture Wearers: a Clinico-microbiological Study. Materia socio-medica. 2017;29(1):26-9.

6. Byrd WC, Schwartz-Baxter S, Carlson J, Barros S, Offenbacher S, Bencharit S. Role of salivary and candidal proteins in denture stomatitis: an exploratory proteomic analysis. Molecular bioSystems. 2014;10(9):2299-304.

7. Moosazadeh M, Akbari M, Tabrizi R. Denture Stomatitis and Candida albicans in Iranian Population: A Systematic Review and MetaAnalysis. J dentistry (Shiraz, Iran). 2016;17(3):28392.

8. Kovac-Kovacic M, Skaleric U. The prevalence of oral mucosal lesions in a population in Ljubljana, Slovenia. J oral pathol. 2000;29(7):331-5.

9. García-Pola Vallejo MJ, Martínez Díaz-Canel AI, García Martín JM, González García M. Risk factors for oral soft tissue lesions in an adult Spanish population. Community dentistry oral epidemiol. 2002;30(4):277-85.

10. Mumcu G, Cimilli H, Sur H, Hayran O, Atalay T. Prevalence and distribution of oral lesions: a crosssectional study in Turkey. Oral diseases. 2005;11(2):81-87.

11. Budtz-Jörgensen E, Stenderup A, Grabowski M. An epidemiologic study of yeasts in elderly denture wearers. Community dentistry and oral epidemiol. 1975;3(3):115-9.

12. Nevalainen MJ, Närhi TO, Ainamo A. Oral mucosal lesions and oral hygiene habits in the home-living elderly. J oral rehabilitation. 1997;24(5):332-7.

13. Mikkonen M, Nyyssönen V, Paunio I, Rajala M. Oral hygiene, dental visits and age of denture for prevalence of denture stomatitis. Community dentistry oral epidemiol. 1984;12(6):402-5.

14. Reichart PA. Oral mucosal lesions in a representative cross-sectional study of aging Germans. Community dentistry oral epidemiol. 2000;28(5):390-8.

15. Vigild M. Oral mucosal lesions among institutionalized elderly in Denmark. Community dentistry and oral epidemiology. 1987;15(6):309-13.

16. Freitas JB, Gomez RS, De Abreu MH, Ferreira EFE. Relationship between the use of full dentures and mucosal alterations among elderly Brazilians. J oral rehabilitation. 2008;35(5):370-4. 
17. Espinoza I, Rojas R, Aranda W, Gamonal J. Prevalence of oral mucosal lesions in elderly people in Santiago, Chile. $\mathrm{J}$ oral pathol med. 2003;32(10):571-5.

18. Gendreau L, Loewy ZG. Epidemiology and Etiology of Denture Stomatitis. J Prosthodontics. 2011;20(4):251-60.

19. Aoun G, Cassia A. Evaluation of denture-related factors predisposing to denture stomatitis in a lebanese population. Materia socio-medica. 2016;28(5):392-6.

20. MacPhail LA, Komaroff E, Alves ME, Navazesh M, Phelan JA, Redford M. Differences in risk factors among clinical types of oral candidiasis in the Women's Interagency HIV Study. Oral surg oral med oral pathol oral radiol endodontics. 2002;93(1):4555 .

21. McMullan-Vogel CG, Jüde HD, Ollert MW, Vogel CW. Serotype distribution and secretory acid proteinase activity of Candida albicans isolated from the oral mucosa of patients with denture stomatitis. Oral microbiol immunol. 1999;14(3):183-9.

22. Carvalho de Oliveira T, Frigerio M, Yamada M, Birman E. Evaluation of denture stomatitis in users of complete dentures. Pesquisa Odontológica Brasileira. 2000;14:219-24.

23. Coco BJ, Bagg J, Cross LJ, Jose A, Cross J, Ramage G. Mixed Candida albicans and Candida glabrata populations associated with the pathogenesis of denture stomatitis. Oral microbiol immunol. 2008;23(5):377-83.

24. Frenkel H, Harvey I, Newcombe RG. Oral health care among nursing home residents in Avon. Gerodontology. 2000;17(1):33-8.

25. Baran I, Nalçacı R. Self-reported denture hygiene habits and oral tissue conditions of complete denture wearers. Arch gerontol geriatrics. 2009;49(2):237-41.

26. Vasconcelos LCD, Vasconcelos LCDS, Ghersel ELDA, Veloso DJ, Cunha Pâsmda. Denture hygiene: importance in denture stomatitis control. RGO Revista Gaúcha de Odontologia. 2013;61(2):255-61.

27. Martins K, Gontijo S. Treatment of denture stomatitis: literature review. Revistas. 2017;74:215.

28. Aoun G, Saadeh M, Berberi A. Effectiveness of Hexetidine $0.1 \%$ Compared to Chlorhexidine Digluconate $0.12 \%$ in Eliminating Candida albicans Colonizing Dentures: A Randomized Clinical In Vivo Study. J int oral health. 2015;7(8):5-8.

29. Sutula J, Coulthwaite L, Thomas L, Verran J. The effect of a commercial probiotic drink on oral microbiota in healthy complete denture wearers. Microbial ecol health disease. 2012;23.

30. Cross LJ, Williams DW, Sweeney CP, Jackson MS, Lewis MA, Bagg J. Evaluation of the recurrence of denture stomatitis and Candida colonization in a small group of patients who received itraconazole. Oral surg oral med oral pathol oral radiol endodontics. 2004;97(3):351-8.

31. Cross LJ, Bagg J, Wray D, Aitchison T. A comparison of fluconazole and itraconazole in the management of denture stomatitis: a pilot study. J dentistry. 1998;26(8):657-64.

32. Arruda CNF, Salles MM, Badaró MM. Effect of sodium hypochlorite and Ricinus communis solutions on control of denture biofilm: A randomized crossover clinical trial. $\mathrm{J}$ prosthetic dentistry. 2017;117(6):729-34.

33. Yousef M, Abdelaziz A, Essa M, Fahmi M. Assessment of Photodynamic Therapy and Miconazole in the Management of Denture Stomatitis. Assessment of Photodynamic Therapy and Miconazole in the Management of Denture Stomatitis. 2018;83-7.

34. Martins KV, De Lacerda Gontijo SM. Treatment of denture stomatitis: literature review. Rev Bras Odontol. 2017;74(3):215-20.

35. Milillo L, Muzio LL, Carlino P, Serpico R, Coccia E, Scully C. Candida-related denture stomatitis: a pilot study of the efficacy of an amorolfine antifungal varnish. Int J Prosthodontics. 2005;18:1.

36. Budtz-Jørgensen E, Holmstrup P, Krogh P. Fluconazole in the treatment of Candida-associated denture stomatitis. Antimicrobial agents chemother. 1988;32(12):1859-63.

37. Martin-Mazuelos E, Aller AI, Romero MJ, et al. Response to fluconazole and itraconazole of Candida spp. in denture stomatitis. Mycoses. 1997;40(78):283-9.

38. Cross LJ, Bagg J, Aitchison TC. Efficacy of the cyclodextrin liquid preparation of itraconazole in treatment of denture stomatitis: comparison with itraconazole capsules. Antimicrobial agents chemother. 2000;44(2):425-7.

39. Friccius H, Pohla H, Adibzadeh M, SiegelsHübenthal P, Schenk A, Pawelec G. The effects of the antifungal azoles itraconazole, fluconazole, ketoconazole and miconazole on cytokine gene expression in human lymphoid cells. Int $\mathrm{j}$ immunopharmacol. 1992;14(5):791-9.

40. Al-Shayyab MH, Abu-Hammad OA, Al-Omiri MK, Dar-Odeh NS. Antifungal prescribing pattern and attitude towards the treatment of oral candidiasis among dentists in Jordan. Int dental j. 2015;65(4):216-6.

41. Nairn RI. Nystatin and amphotericin B in the treatment of denture-related candidiasis. Oral surg oral med and oral pathol. 1975;40(1):68-75.

42. Falah-Tafti A, Jafari AA, Lotfi-Kamran MH, Fallahzadeh H, Hayan RS. A Comparison of the eFficacy of Nystatin and Fluconazole Incorporated into Tissue Conditioner on the In Vitro Attachment and Colonization of Candida albicans. Dental res $\mathrm{j}$. 2010;7(1):18-22.

43. Geerts GA, Stuhlinger ME, Basson NJ. Effect of an antifungal denture liner on the saliva yeast count in patients with denture stomatitis: a pilot study. J oral rehabilitation. 2008;35(9):664-9.

44. Mahdavi Omran S, Rezaei Dastjerdi M, Zuashkiani M, Moqarabzadeh V, Taghizadeh-Armaki M. In Vitro Antifungal Susceptibility of Candida species 
Isolated from Iranian Patients with Denture Stomatitis. BioMed research int. 2018;2018:3086586.

45. Khozeimeh F, Shahtalebi MA, Noori M, Savabi O. Comparative evaluation of ketoconazole tablet and topical ketoconazole $2 \%$ in orabase in treatment of Candida-infected denture stomatitis. J Contemp Dent Pract. 2010;11(2):017-24.

46. Kadir T, Gümrü B, Uygun-Can B. Phospholipase activity of Candida albicans isolates from patients with denture stomatitis: the influence of chlorhexidine gluconate on phospholipase production. Arch oral biol. 2007;52(7):691-6.

47. Maciel CM, Piva MR, Ribeiro MA, De Santana Santos T, Ribeiro CF, Martins-Filho PR. Methylene Blue-Mediated Photodynamic Inactivation Followed by Low-Laser Therapy versus Miconazole Gel in the Treatment of Denture Stomatitis. J prosthodontics. 2016;25(1):28-32.

48. De Figueiredo Freitas LS, Rossoni RD, Jorge AOC, Junqueira JC. Repeated applications of photodynamic therapy on Candida glabrata biofilms formed in acrylic resin polymerized. Lasers med sci. 2017;32(3):549-55.

49. Mima EG, Pavarina AC, Ribeiro DG, Dovigo LN, Vergani CE, Bagnato VS. Effectiveness of photodynamic therapy for the inactivation of Candida spp. on dentures: in vitro study. Photomedicine laser surg. 2011;29(12):827-33.

50. Acosta-Torres LS, Mendieta I, Nuñez-Anita RE, Cajero-Juárez M, Castaño VM. Cytocompatible antifungal acrylic resin containing silver nanoparticles for dentures. Int $\mathrm{j}$ nanomed. 2012;7:4777-86.

Cite this article as: Bukhari MA, Algahtani MA, Alsuwailem FA, Alogaiel RM, Almubarak SH, Alqahtani SS et al. Epidemiology, etiology, and treatment of denture stomatitis. Int J Community Med Public Health 2022;9:981-6. 